\title{
PARP target practice
}

\section{By Joanne Kotz, Senior Editor}

Swedish researchers have used an in vitro screen to evaluate the target selectivity of a panel of small molecule poly(ADP-ribose) polymerase inhibitors. ${ }^{1}$ The findings could eventually lead to next-generation compounds with a better therapeutic index than those now in the clinic for cancer.

The 17 human poly(ADP-ribose) polymerase (PARP) enzymes attach poly-ADP-ribose or mono-ADP-ribose to target proteins. The

Figure 1. PARP inhibitors in profile. A Swedish research team has profiled the selectivity of a panel of poly(ADP-ribose) polymerase (PARP) inhibitors, including three inhibitors in Phase II trials for cancer-olaparib (AZD2281) from AstraZeneca plc, veliparib (ABT888) from Abbott Laboratories and rucaparib (CO-338) from

Clovis Oncology Inc., Pfizer Inc. and Cancer Research UK.

The team used an in vitro assay that quantified binding, a surrogate measure for inhibitor potency, of 185 PARP inhibitors to 13 of the 17 human PARP enzymes, including the tankyrases, TNKS1 and TNKS2.

In the heat map, red indicates strong binding, orange signifies intermediate binding and yellow represents weak binding. These binding measurement ranges correspond to $\mathrm{IC}_{50}$ values of approximately low to mid nanomolar, mid to high nanomolar and high nanomolar to low micromolar, respectively. Gray indicates no detectable binding.

Of the three clinical PARP inhibitors profiled, veliparib was the most selective, binding strongly to PARP1-3, and rucaparib the most promiscuous, binding detectably to nine PARP domains. best-characterized PARP family members are the DNA repair enzymes PARP1 and PARP2. The tankyrases, TNSK1 and TNKS2, are members of the PARP family that are involved in the DNA damage response as well as regulation of wingless-type MMTV integration site (WNT) signaling. The function of many of the remaining PARP enzymes is poorly understood.

Clinical interest in PARPs heated up in 2005 when two back-toback papers in Nature showed that PARP inhibitors selectively killed cells and tumors with deficiencies in the DNA repair enzymes breast cancer 1 early onset (BRCA1) or BRCA2. ${ }^{2,3}$ Loss-of-
"We don't yet know if a pan-PARP inhibitor versus a more selective PARP inhibitor is better therapeutically and in which contexts inhibitors with different selectivity profiles may have value." -Paul Workman,

The Institute of Cancer Research function genetic mutations in $B R C A 1$ or BRCA2 occur in multiple cancers, notably breast and ovarian.

At least three PARP inhibitors are in Phase II testing in various cancers-olaparib (AZD2281) from AstraZeneca plc, veliparib (ABT888) from Abbott Laboratories and rucaparib (CO-338) from Clovis Oncology Inc., Pfizer Inc. and Cancer Research UK.

In December 2011, AstraZeneca discontinued development of olaparib for serous ovarian cancer based on an interim analysis of Phase II data suggesting the molecule would not lead to a sufficient overall survival benefit.

Although many first-generation PARP inhibitors were developed against PARP1, the specificity of the inhibitors for PARP1 over other PARP family members has not been systematically studied. A team led by Herwig Schüler thus set out to profile the selectivity of these PARP inhibitors.

At the time of the study, Schüler was a principal investigator at the Structural Genomics Consortium site in Stockholm. That site has since been shuttered, and Schüler is now a principal investigator in the Department of Medical Biochemistry and Biophysics at the Karolinska Institute.

First, the Swedish team expressed and purified the catalytic domains from 13 of the 17 human PARP enzymes. The team then established an in vitro assay for measuring ligand binding to the domains, a surrogate measure for inhibitor potency that was used because enzymatic assays are not available for all of the PARP enzymes.

Using this assay, the researchers quantified the binding of 185 known and potential PARP inhibitors to the 13 PARP domains. The vast majority of the molecules were promiscuous, binding to multiple PARP family members.

Of the three clinical PARP inhibitors profiled, veliparib was the most selective, binding strongly to PARP1-3 and detectably to two additional PARP family members. Olaparib bound strongly to PARP1-4 and weakly to three additional PARP enzymes. Rucaparib was the most promiscuous-binding strongly to PARP1-4 and TNKS1, as well as 


\section{ANALYSIS}

\section{TARGETS \& MECHANISMS}

more weakly to four additional PARP family members including TNKS2 (see Figure 1, "PARP inhibitors in profile").

Results were published in Nature Biotechnology.

\section{The selectivity difference}

The Nature Biotechnology paper is "an important first systematic profiling study, and the selectivity of PARP inhibitors for the different

"These are interesting data but binding data only. We obviously will be pursuing this to further characterize our molecule [rucaparib] to see if it is truly inhibitory for TNKS1 and TNKS2 and whether that imparts additional utility to the molecule as a cancer therapeutic."

-Andrew Allen, Clovis Oncology Inc. family members could be extremely important in terms of their use as chemical tools and drugs," said Paul Workman, deputy CEO and director of the Cancer Research UK Cancer Therapeutics Unit at The Institute of Cancer Research.

"We don't yet know if a pan-PARP inhibitor versus a more selective PARP inhibitor is better therapeutically and in which contexts inhibitors with different selectivity profiles may have value. For example, hitting the tankyrases as well as PARP 1-4 could have therapeutic significance but could also lead to side effects," Workman added.

Indeed, a key question now for Clovis is whether the broader selectivity profile of its compound will translate into a better therapeutic index than the more selective veliparib and olaparib.

"These are interesting data but binding data only. We obviously will be pursuing this to further characterize our molecule [rucaparib] to see if it is truly inhibitory for TNKS1 and TNKS2 and whether that imparts additional utility to the molecule as a cancer therapeutic," said Andrew Allen, EVP of clinical and preclinical development and CMO of Clovis.

Allen cited results published by Novartis AG in Nature in 2009 that inhibiting TNKS1 and TNKS2 antagonizes WNT signaling. ${ }^{4} \mathrm{He}$ added that WNT signaling is a "validated target, most classically in colorectal cancer but also in additional cancers including breast and melanoma."

Thus, Allen said that Clovis will now be looking preclinically to see if inhibiting tankyrases in combination with inhibiting PARP1 and PARP2 could be synergistic in breast cancer patients with BRCA mutations or in a different cancer patient population. Alternatively, tankyrase inhibition could open up new cancer indications for the molecule.

The company will investigate these possibilities by comparing rucaparib to clinical-stage PARP inhibitors with distinct binding profiles, particularly AstraZeneca's olaparib, in in vitro enzymatic assays, cell models and animal models, Allen added.

Alternatively, rucaparib could have greater toxicity than more selective PARP inhibitors. "We don't know enough to know whether tankyrase inhibition could be a liability. We haven't seen anything obvious, but whether we will see a tox profile that looks subtly different from other PARP inhibitors remains to be seen," said Allen.

\section{Getting particular}

Next up for the Swedish team will be developing selective inhibitors as tools to clarify the function and therapeutic potential of particular PARP enzymes, said Schüler.

Schüler said the team is "trying to not overlap too much with what we believe will be targeted by pharmaceutical companies," which he said would likely be PARP1-4 and the tankyrases.

Instead, the team is developing inhibitors against three of the other PARP family members. Although Schüler did not disclose which PARP enzymes are being targeted, potential chemical starting points for developing selective inhibitors against PARP10, PARP14 and PARP16 are discussed in the paper.

The results reported in Nature Biotechnology are not patented.

Kotz, J. SciBX 5(13); doi:10.1038/scibx.2012.323

Published online March 29, 2012

\section{REFERENCES}

1. Wahlberg, E. et al. Nat. Biotechnol.; published online Feb. 19, 2012; doi:10.1038/nbt.2121

Contact: Herwig Schüler, Karolinska Institute, Stockholm, Sweden e-mail: herwig.schuler@ki.se

2. Bryant, H.E. et al. Nature 434, 913-917 (2005)

3. Farmer, H. et al. Nature 434, 917-921 (2005)

4. Huang, S.-M.A. et al. Nature 461, 614-620 (2009)

COMPANIES AND INSTITUTIONS MENTIONED

Abbott Laboratories (NYSE:ABT), Abbott Park, III. AstraZeneca plc (LSE:AZN; NYSE:AZN), London, U.K. Cancer Research UK, London, U.K. Clovis Oncology Inc. (NASDAQ:CLVS), Boulder, Colo. The Institute of Cancer Research, London, U.K. Karolinska Institute, Stockholm, Sweden Novartis AG (NYSE:NVS; SIX:NOVN), Basel, Switzerland Pfizer Inc. (NYSE:PFE), New York, N.Y.

Structural Genomics Consortium, Stockholm, Sweden 